\title{
Primeros datos de reproducción confirmada de Anax ephippiger (Burmeister, 1839) (Odonata: Aeshnidae) en País Vasco y Navarra.
}

\section{First records of confirmed reproduction of Anax ephippiger (Burmeister, 1839) (Odonata: Aeshnidae) in the Basque Country and Navarre.}

Isabel Garnika1, Elena Artika²

\section{Resumen}

El hallazgo de un ejemplar inmaduro en agosto de 2017 en un carrizal en Navarra, y de varias exuvias de Anax ephippiger en septiembre del mismo año en un estanque urbano en Álava (País Vasco), confirman la primera reproducción de esta especie en Navarra y en el País Vasco, respectivamente.

Palabras clave: Odonata, Anax ephippiger, reproducción, inmaduro, exuvia, Navarra, País Vasco, España.

\section{Abstract}

The discovery of a teneral specimen in a reed bed in Navarre in August 2017 and of several exuviae of Anax ephippiger in an urban pond in Álava (Basque Country) in September 2017, confirm the first reproduction of this species in Navarre and in the Basque Country, respectively.

Key words: Odonata, Anax ephippiger, reproduction, teneral, exuvia, Navarre, Basque Country, Spain.

\footnotetext{
1 Plaza Santa Bárbara, 6 - 5 I. 01004 Vitoria-Gasteiz

Correspondencia: isabelgarnika@gmail.com

2 Calle Alfonso el Batallador, 5 bis - 4A. 31007 Pamplona
} 


\section{Laburpena}

2017ko abuztuan ale heldugabe baten aurkikuntzak Nafarroko lezkadi batean, eta urte bereko iralean Anax ephippiger-en exubia batzuenak hiri urmael batean Araban (Euskadi), espezie honen lehenengo ugalketa baieztatzen dute Nafarroan eta Euskadin, hurrenez hurren.

Gako hitzak: Odonata, Anax ephippiger, ugalketa, heldugabe, exubia, Nafarroa, Euskadi, Espainia.

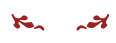

Anax ephippiger (Burmeister, 1839) es la más pequeña de las especies de Anax presentes en Europa, y a menudo se clasifica en el género Hemianax (Boudot, 2013). Sus ojos son pardos en la parte superior, y el color del abdomen es marrón claro. Los machos presentan una característica mancha azul en el segundo segmento abdominal que, al contrario que en Anax parthenope (Selys, 1839), se limita a la parte dorsal y no se extiende a los laterales (Dijkstra \& Lewington, 2006).

Se trata de una especie afrotropical propia de ambientes áridos a semiáridos, que también ocupa el Sudoeste Asiático, la Península Arábiga e India (Lambret \& Boudot, 2013). Se reproduce en aguas temporales que llegan a secarse la mayoría de los veranos, generalmente en áreas con estaciones secas y de lluvias muy marcadas. La especie presenta una tendencia dispersiva muy acentuada, que le hace abandonar su hábitat de reproducción a edades muy tempranas y cubrir grandes distancias en sus desplazamientos (Boudot \& Kalkman, 2015).

Los ciclos y migraciones estacionales de la especie, que se resumen a continuación, han sido descritos por Lambret \& Boudot (2013). En África, el periodo de puesta comienza durante la estación lluviosa, que se extiende de mayo o junio hasta septiembre, y las emergencias masivas se producen entre finales de agosto y noviembre, según los años. Posteriormente, altas concentraciones de $A$. ephippiger se detienen en invierno al sur del Atlas en el Magreb. En primavera, estos individuos, ya en un estado maduro, reanudan su movimiento hacia el norte y esta segunda ola migratoria llega a Europa después de cruzar el Mediterráneo, en números muy variables de año a año.

La mayoría de los lugares de reproducción en Europa se encuentran en humedales costeros (Kalkman, 2010), sobre todo en el entorno mediterráneo, principalmente en aguas estancadas y someras con una temperatura elevada del agua que permite el rápido desarrollo larvario (Boudot \& Kalkman, 2015). El devenir de estas generaciones europeas todavía no está claro; existen indicios que hacen plausible el retorno exitoso al núcleo de su área original de distribución, cruzando el Mediterráneo y/o las regiones montañosas de Turquía, pero para los individuos que permanecen o siguen migrando a través de Europa, no hay virtualmente posibilidades de reproducción (Lambret \& Boudot, 2013). Según Boudot \& Kalkman (2015), únicamente se conocen dos regis- 
tros de supervivencia de larvas durante el invierno, concretamente en el Parque Nacional de Doñana (España) en 1979 y en el Delta del Ródano (Francia) en 2000.

A. ephippiger se considera en España como una especie ligada a los ambientes más cálidos del área meridional y de la costa mediterránea, estando presente en 35 de las 47 provincias de la España peninsular (Prunier et al. 2015). En Portugal, Maravalhas \& Soares (2013) indican por su parte que algunos años llega a ser una especie abundante, y señalan su presencia en toda la zona litoral del país y algunos puntos del interior.

En la mitad norte de la Península Ibérica se ha observado actividad reproductora de la especie en diversas ocasiones, pero únicamente se ha confirmado la emergencia de individuos en Galicia, donde en septiembre de 2014 se detectó la presencia de un ejemplar inmaduro de A. ephippiger en Comarca do Barbanza (Vilas Souto, 2014), y en Cataluña, donde se han encontrado numerosas exuvias en diferentes años en el estany d'Ivars i Vila-sana en el sur de Lérida (Martín et al., 2016).

En el País Vasco, las primeras citas de A. ephippiger tuvieron lugar en 2009 en Álava (fig. 1): diversas observaciones, incluidas cópulas y puestas, en el embalse de Ullibarri-Ganboa entre el 12 y el 31 de mayo, y dos machos el día 16 del mismo mes

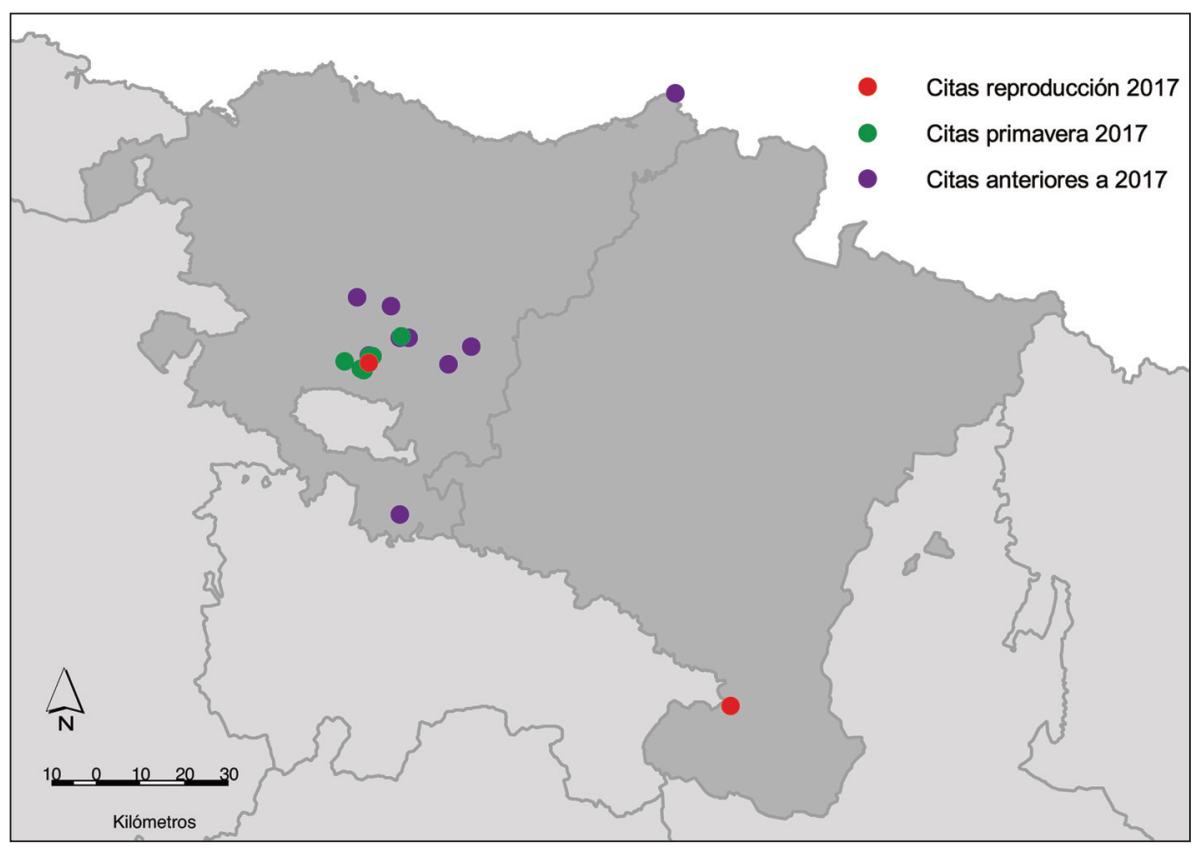

Fig. 1.- Situación de las citas de Anax ephippiger en País Vasco y Navarra. En la referencia a la cita navarra recogida en Prunier et al. (2015) no figura ninguna localidad, por lo que no se ha incluido en el mapa.

Fig. 1.- Location of Anax ephippiger records in the Basque Country and Navarre. The location of the Navarre record in Prunier et al. (2015) is not specified, so it is not included in the map. 
en la laguna de Musco en Laguardia (Gainzarain, 2010). De nuevo en Álava, durante los meses de abril y mayo de 2011 se observaron cópulas y puestas en la balsa de Ordoñana, los embalses de Ullibarri y Urrunaga, y en la balsa de Betoño en Salburua. Sin embargo, ni en 2009 ni en 2011 se llegó a comprobar la emergencia de ningún ejemplar (Gainzarain et al., 2013).

A. ephippiger también ha sido registrada en Gipuzkoa, datando la primera cita, que también lo es para el conjunto de la cornisa cantábrica, de mayo de 2011 en el Parque de Plaiaundi (Mezquita-Aranburu \& Ocharan, 2012).

En 2017, se registró de nuevo la presencia de numerosos ejemplares de $A$. ephippiger en diferentes puntos de la Llanada Alavesa, concretamente los días 15, 16, 17 y 19 de marzo. Así en la plataforma Ornitho (www.ornitho.eus) se recogen 14 observaciones, con 27 ejemplares en total, en diversos humedales del entorno de VitoriaGasteiz y también en el embalse de Ullibarri.

La reproducción exitosa de la especie en el País Vasco se confirmó en el curso de uno de los muestreos del programa de seguimiento de odonatos que se lleva a cabo en Vitoria-Gasteiz desde 2014 en el seno de los proyectos de ciencia ciudadana del Centro de Estudios Ambientales del ayuntamiento de la ciudad (Radaelli et al., 2016). La observación tuvo lugar concretamente el 01.09.2017, cuando se localizó un ejemplar inmaduro y se recogieron diez exuvias (fig. 2) entre la vegetación del estanque del Parque del Este de Vitoria-Gasteiz (UTM 30T 528443, 4743729). Situado a 525 m s.n.m., este estanque se localiza en un entorno urbano y está formado por un vaso de hormigón rodeado en parte de césped, con una lámina de agua de aproximadamente $1.180 \mathrm{~m}^{2}$ y una profundidad máxima de $45 \mathrm{~cm}$. Ha sido naturalizado recientemente (octubre 2016), instalando en 300 metros de orilla biorrollos sumergidos con vegetación palustre (sobre todo Acorus calamus L., Iris pseudacorus L., y Scirpus lacustris L.) y cantos rodados, así como dos pequeñas isletas interiores también revegetadas. La identificación se realizó verificando que las exuvias halladas en el estanque presentaban los siguientes caracteres diagnósticos que las diferencian de las otras especies ibéricas de $\operatorname{Anax}(A$. imperator (Leach, 1815) y A. parthenope): la unión prementum/postmomentum del labium no llega más allá del segundo par de patas, y que los machos carecen de la expansión al final del abdomen (Butler, 1998). Del mismo modo, las longitudes de las exuvias, que para esta especie debe estar comprendida entre los 42 y 45 mm según Doucet (2011) y entre los 42 y 46 mm según Grand \& Boudot (2006), variaron entre 42 - 45 mm con excepción de un ejemplar anómalo que ha alcanzado una longitud de $50 \mathrm{~mm}$. Las exuvias de las otras especies de Anax mencionadas anteriormente tienen una longitud superior a los $45 \mathrm{~mm}$ según Doucet (2011) y a los 47 mm según Grand \& Boudot (2006).

En cuanto a Navarra, según Prunier et al. (2015), únicamente se conocía una cita de A. ephippiger hasta 2015. A pesar de no haber podido confirmar la presencia de esta 


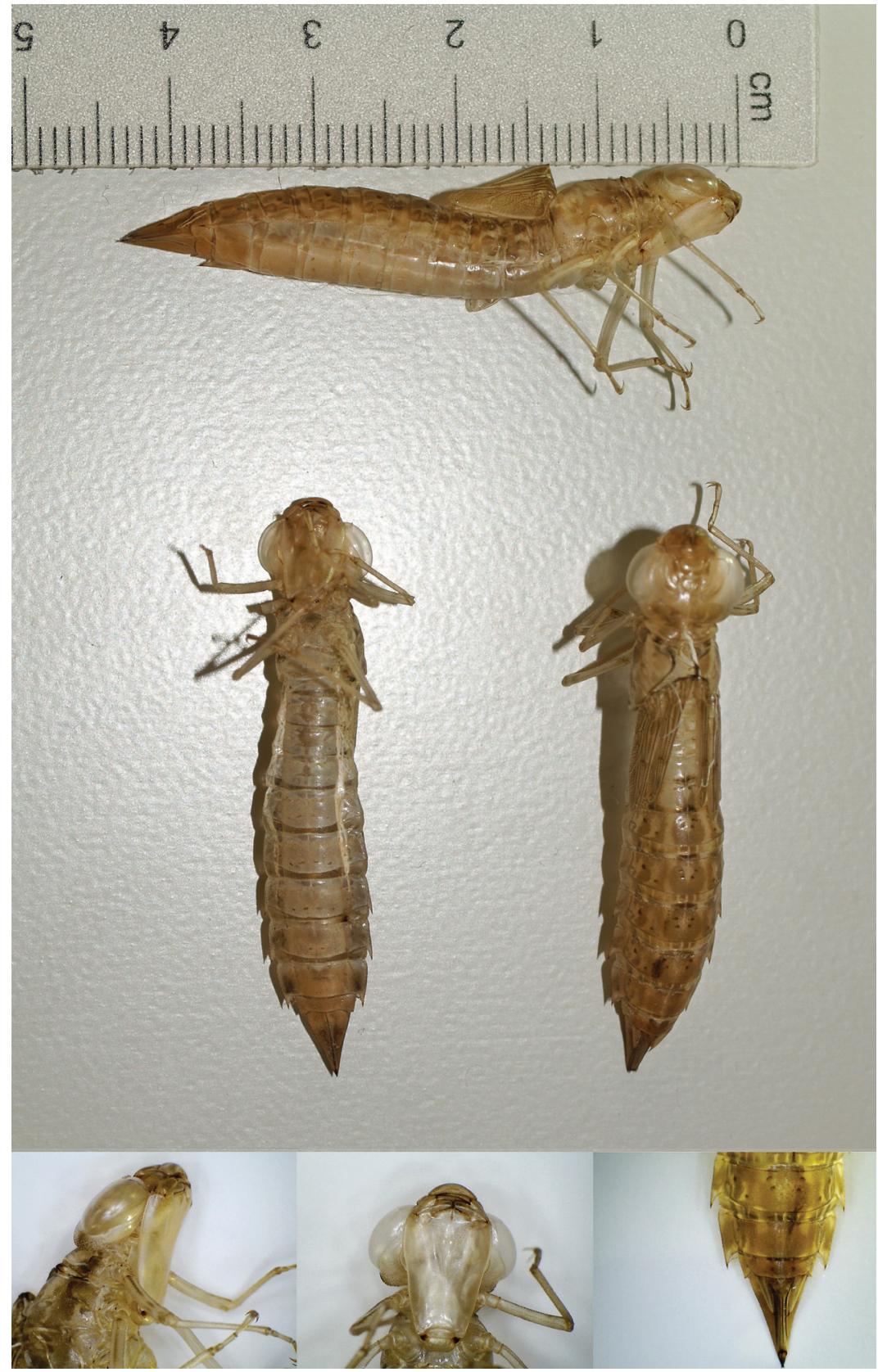

Fig. 2.- Exuvias de Anax ephippiger encontradas en un estanque urbano de Vitoria-Gasteiz (Álava).

Fig. 2.- Anax ephippiger exuviae found in an urban pond in Vitoria-Gasteiz (Alava). 
especie durante la primavera, el 27.08.2017 se halló un ejemplar inmaduro de A. ephippiger (fig. 3) en el Barranco de Valdelafuente, al norte del término municipal de Tudela (Navarra) (UTM 30T 610096, 4666392). Situado a 299 m s.n.m., es uno de los pequeños humedales anexos al río Ebro que forma parte de la Zona de Especial Conservación (ZEC ES2200040) "Río Ebro".

Se encuentra ubicado en un área dominada por extensos cultivos de regadío y de secano en las zonas llanas, y por pastos y matorrales esclerófilos en las laderas. Resulta de la confluencia de otros dos barrancos que son atravesados por el canal de Lodosa y del cual reciben agua.

En su parte más húmeda, la vegetación dominante es la conformada por carrizo Phragmites australis (Cav.) Trin. ex Steudel, acompañada por caña brava Arundo

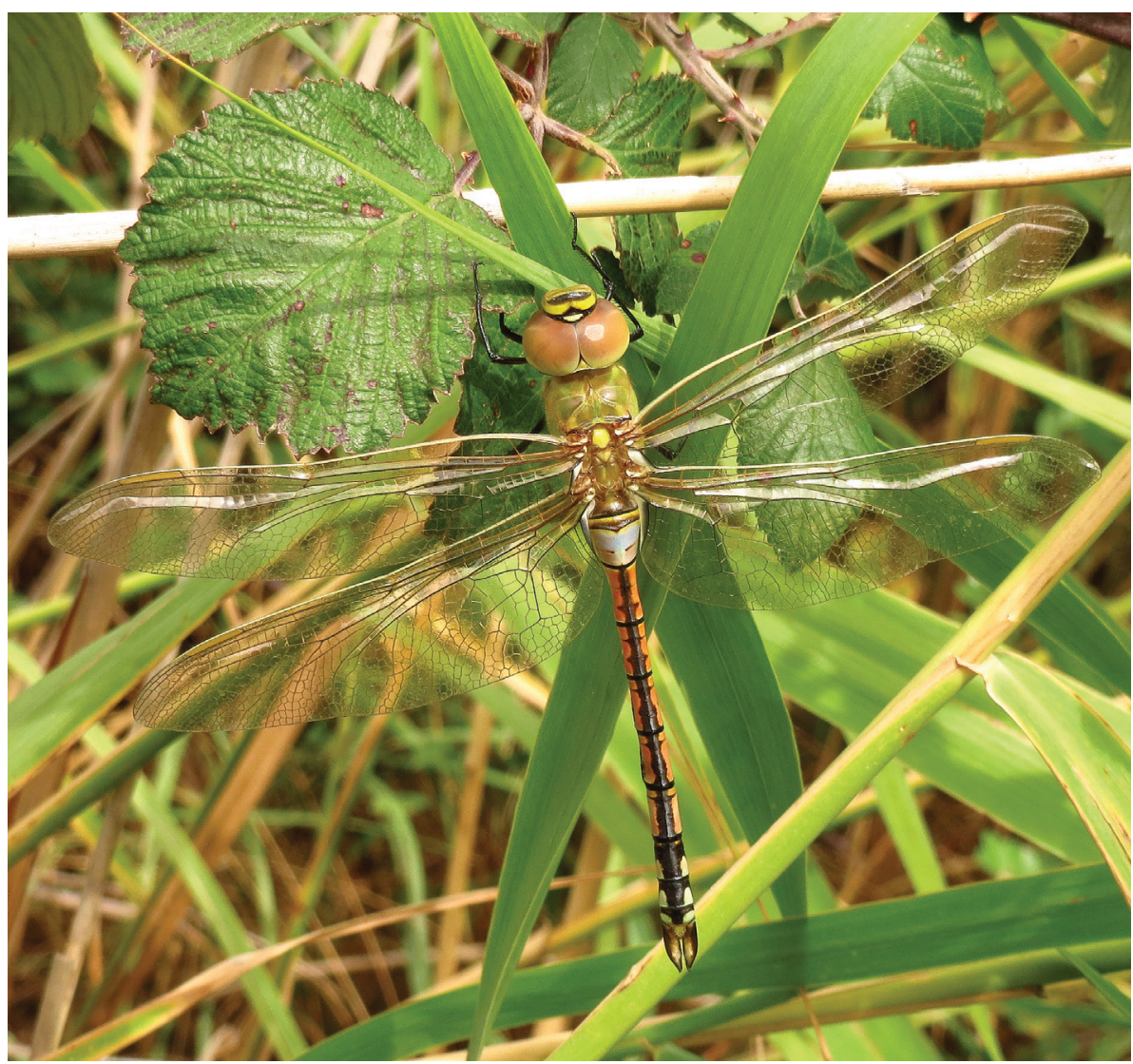

Fig. 3.- Ejemplar inmaduro de Anax ephippiger fotografiado en el Barranco de Valdelafuente en Tudela (Navarra).

Fig. 3.- Anax ephippiger teneral photographed in the Valdelafuente Ravine in Tudela (Navarre). 
donax L., y algunos sauces Salix sp. En ciertos puntos se forman intermitentes zonas encharcadas con escasa vegetación herbácea, juncos Juncus sp., espadañas Typha sp. y algún tamariz Tamarix canariensis Willd.

Estas observaciones confirman la reproducción en el País Vasco y en Navarra de A. ephippiger, un fenómeno registrado hasta ahora muy rara vez en la mitad norte peninsular. Los individuos recién emergidos son con toda probabilidad descendientes de aquellos observados durante el mes de marzo. El rápido desarrollo de las larvas en condiciones climáticas y de hábitat adecuadas permiten la emergencia de una segunda generación al final del verano, tal y como ha sucedido en esta ocasión.

Si bien Jacquemin \& Boudot (1999) estiman un periodo de desarrollo larval de dos a tres meses para esta especie en los hábitats de reproducción de Marruecos que están disponibles por un corto tiempo, también mencionan que, en invierno, con un clima más severo, este periodo se puede ampliar a cuatro o cinco meses. En el caso que aquí se describe, desde la aparición de los individuos de $A$. ephippiger en marzo y el hallazgo de las exuvias y del ejemplar inmaduro transcurren entre 166 y 171 días, tiempo cercano al señalado para un desarrollo larval con temperaturas moderadas.

La llegada y reproducción de esta especie en el norte peninsular han podido verse beneficiadas por las favorables condiciones meteorológicas del año 2017. A nivel nacional, los datos ofrecidos por la Agencia Estatal de Meteorología AEMET (www.aemet.es), muestran un incremento de las temperaturas medias estacionales en los últimos años (2013-2017), con la mayoría de primaveras y veranos con un carácter cálido o muy cálido en comparación con las medias estacionales en el periodo 1981-2010. La primavera de 2017 en concreto ha sido extremadamente cálida, con $1,7^{\circ} \mathrm{C}$ por encima de la media de la estación en el periodo 1981-2010, tratándose además de la primavera más cálida desde 1965. El verano ha tenido asimismo un carácter muy cálido, con $1,6^{\circ} \mathrm{C}$ por encima de la media de la estación en el periodo 1981-2010, siendo el segundo verano más cálido desde 1965.

El aumento de temperaturas en Europa se refleja en la expansión hacia el norte de numerosas especies de odonatos (Boudot \& Kalkman, 2015), siendo previsible que en un futuro próximo el cambio climático haga más frecuentes en latitudes más norteñas los eventos de reproducción de una especie de origen afrotropical como es A. ephippiger.

\section{Agradecimientos}

A José Antonio Gainzarain por su gran ayuda en la redacción de esta nota, a Luis Lobo por poner a nuestra disposición información relativa a las intervenciones llevadas a cabo en el Parque del Este, y a Alberto Castro, David Outomuro y un revisor anónimo por sus valiosas aportaciones al manuscrito original. 


\section{Bibliografía}

Boudot, J.P. 2013. Hemianax versus Anax ephippiger (Burmeister, 1839) (Odonata: Anisoptera: Aeshnidae). Martinia. Hors-série, Hemianax ephippiger - migration 2011: 3-11.

Boudot, J.P., Kalkman, V.J. 2015. Atlas of the European Dragonflies and Damseflies. KNNV Publishing. Zeist, The Netherlands.

Butler, S.G. 1998. The larvae of the european Aeshnidae (Anisoptera). Odonatologica, 27(1): $1-23$.

Dijkstra K. -D.B., Lewington, R. 2006. Guía de Campo de las Libélulas de España y de Europa. Ediciones Omega. Barcelona.

Doucet, G. 2011. Clé de détermination des exuvies des Odonates de France. Societé Française d'Odonatologie. Bois d'Arcy.

Gainzarain, J. A. 2010. Primeras citas de Hemianax ephippiger (Burmeister, 1839) (Odonata: Aeshnidae) y Onychogomphus costae (Sélys, 1885) (Odonata: Gomphidae) para el País Vasco (norte de España). Bol. Soc. Entomol. Aragonesa 46: 525- 526.

Gainzarain, J.A., Ocharan, F.J., Mezquita, I. 2013. Catálogo de los odonatos (Insecta: Odonata) de Álava, norte de España. Bol. Soc. Entomol. Aragonesa 53: 173-185.

Grand, D., Boudot, J.-P. 2006. Les Libellules de France, Belgique et Luxembourg. Biotope, Mèze.

Jacquemin, G., Boudot, J.-P. 1999. Les Libellules (Odonates) du Maroc. Société Française d'Odonatologie. Bois d'Arcy.

Kalkman, V.J. 2010. Anax ephippiger. The IUCN Red List of Threatened Species: e.T59811A12041658. [descargado el 7 de septiembre de 2017].

Lambret P., Boudot J.P. 2013 Hemianax ephippiger (Burmeister, 1839) (Odonata, Anisoptera: Aeshnidae): présentation générale. Martinia. Hors-série, Hemianax ephippiger - migration 2011: 13-27.

Maravalhas, E, Soares, A. 2013. As Libélulas de Portugal. The dragonflies of Portugal. Booky Publisher. Vila Nova da Telha.

Martín, R., Maynou, X., Lockwood, M., Luque, P., Garrigós, B., Vilasis, D., Escolà, J., GarcíaMoreno, J., Oliver, X., Battle, R. M., Palet, J., Sesma, J. M., Rodríguez, M., Müller, P., Piella, L. I. 2016. Les libèl/Iules de Catalunya. Brau Edicions, Figueras.

Mezquita-Aranburu, I., Ocharan, F.J. 2012. Odonatos de Gipuzkoa. Munibe, Cienc. nat. 60:51-75.

Prunier, F., Brotóns, M., Cabana, M., Campos, F., Casanueva, P., Chelmick, D., Cordero Rivera, A., Díaz Martínez, C., Evangelio, J.M., Gainzarain, J.A., García-Moreno,J., Lockwood, M., de los Reyes, L., Mañani, J., Mezquita-Aramburu, I. Muddeman, J., Ocharan, F. J., Otero Pérez, F., Prieto-Lillo, E., Requena, C., Ripoll, J., Rodríguez Luque, F., Rodríguez, P., Romeos, A., Salcedo, J., Vilariño, V. S., Sánchez Balibrea, J., Tamajón Gómez, R., Torralba-Burrial, A., Tovar, C., Winter, P., Zaldívar, R. 2015. Actualización del inventario provincial de Odonatos de España peninsular e Islas Baleares. Boletín ROLA 6: 59-84. 
Radaelli, P., Barreal, S., Belamendia, G. 2016. Campañas de ciencia ciudadana en el Anillo Verde de Vitoria-Gasteiz. Quercus 360: 38-46.

Vilas Souto, J. 2014. Primeros datos de reproducción de Anax ephippiger (Burmeister, 1839) en Galicia (Odonata: Aeshnidae). BV News Publicaciones Científicas 3: 91-93. 